Supporting Information

\title{
Polymer Solid Phase Grafting at Temperature Higher than Polymer Melting Point through Selective Heating
}

Songhe Wang ${ }^{1,2}$, Xiaohong Zhang ${ }^{2}$, Chao Jiang², Haibin Jiang 2 , Yujing Tang 2 , Juan

$\mathrm{Li}^{2}$, Minqiao Ren ${ }^{2}$, Jinliang Qiao ${ }^{* 1,2}$

${ }^{1}$ College of Materials Science and Engineering, Beijing University of Chemical

Technology, Beijing 100029, (China).

${ }^{2}$ SINOPEC Beijing Research Institute of Chemical Industry, Beijing 100013, (China).

*Corresponding Author: qiaojl.bjhy@sinopec.com 

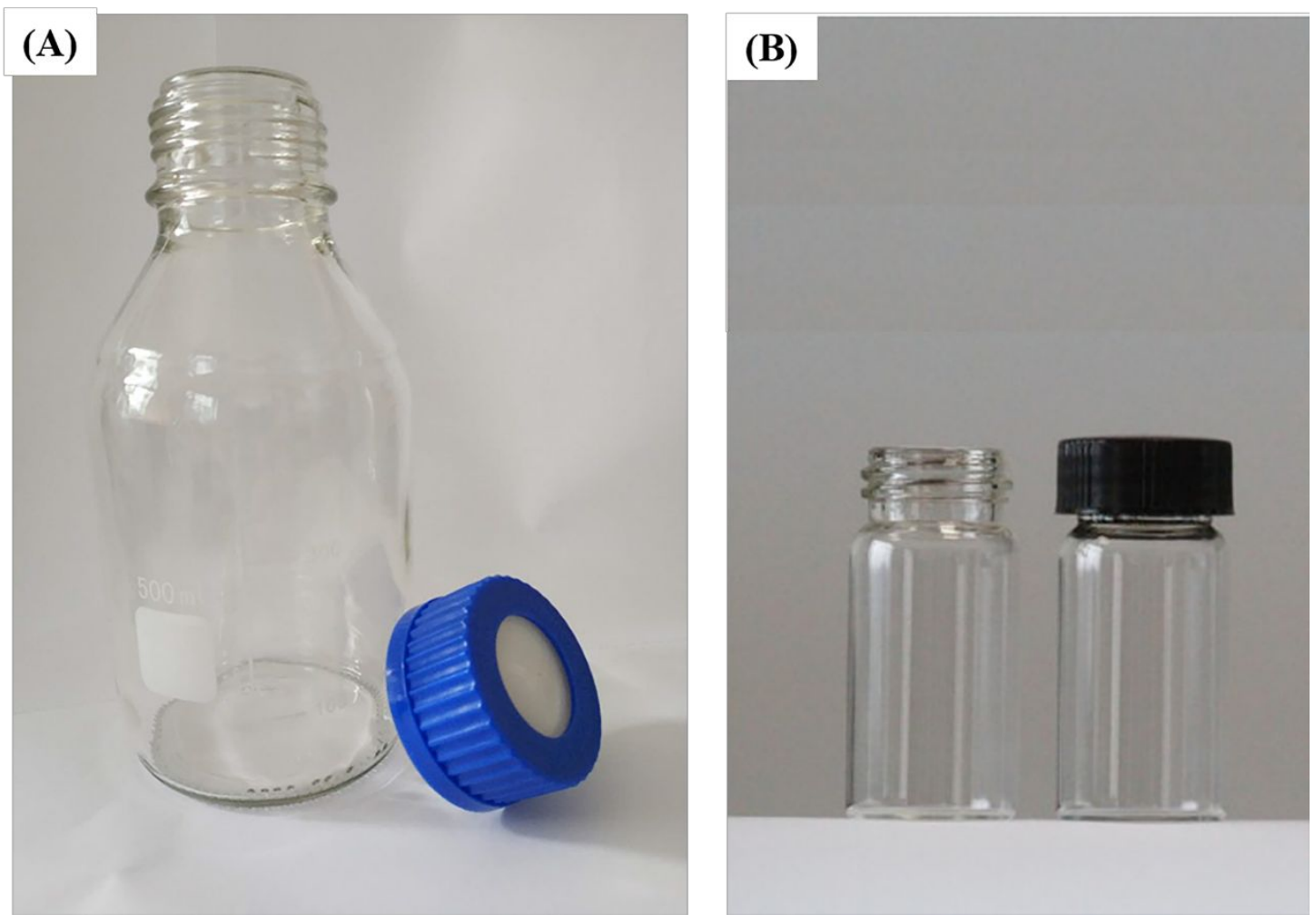

Figure S1. Reaction flasks for microwave grafting(A) and temperature measurement of $\mathrm{MAH}$ and $\mathrm{NaCl}(\mathrm{B})$.
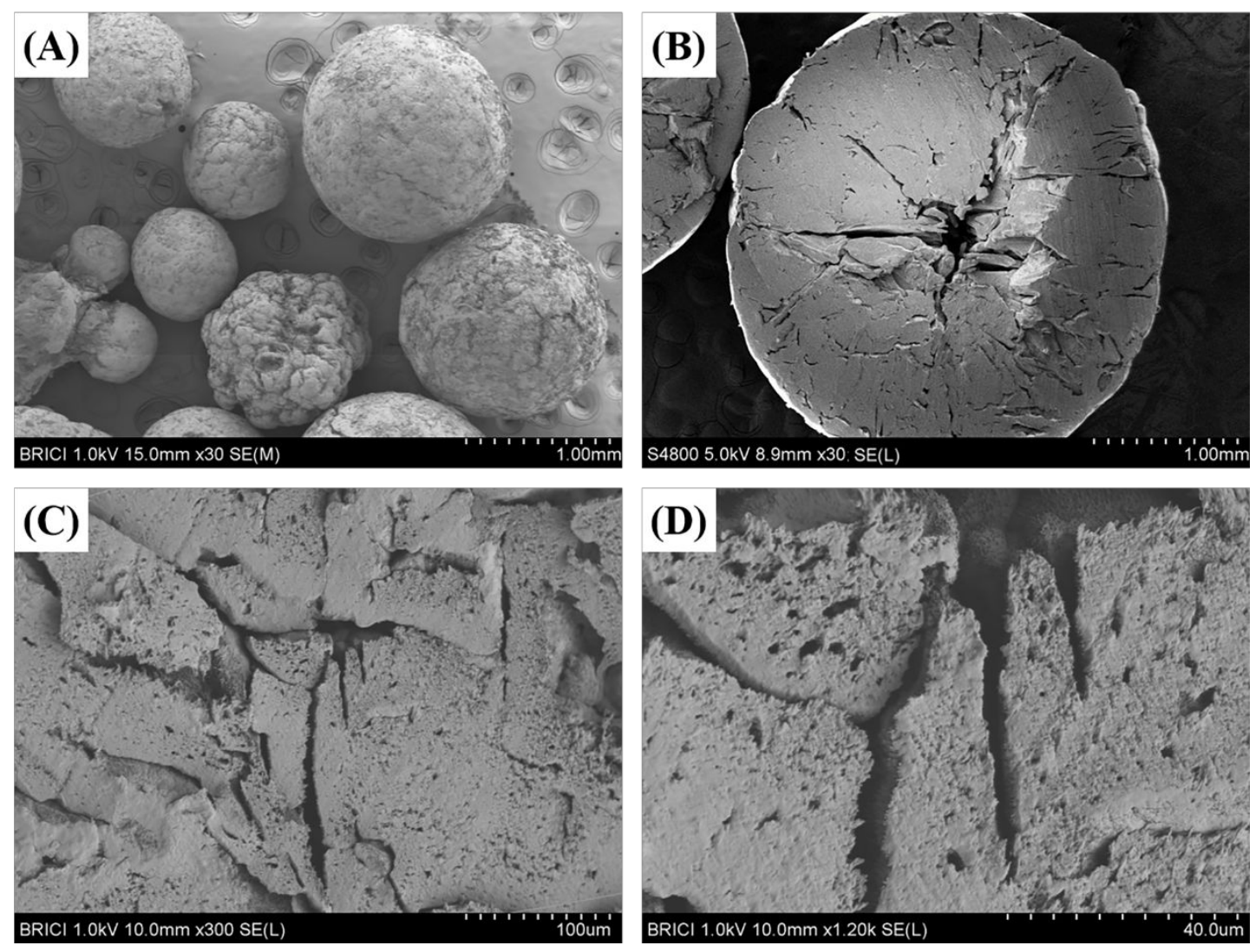

Figure S2. SEM images of the surface and internal pores of PP spherical powder. 
(A)

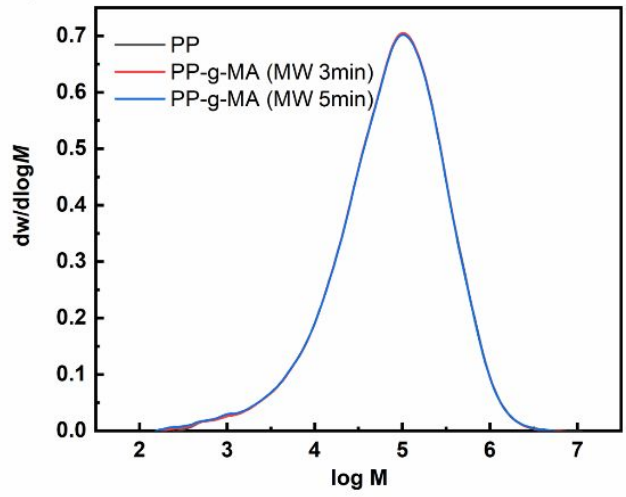

(C)

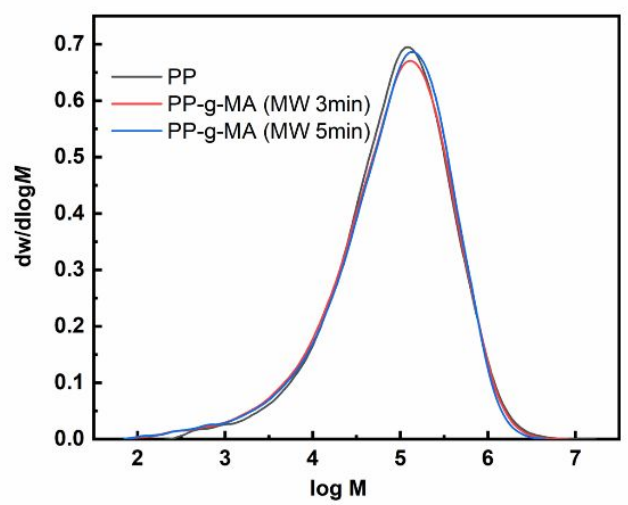

(B)

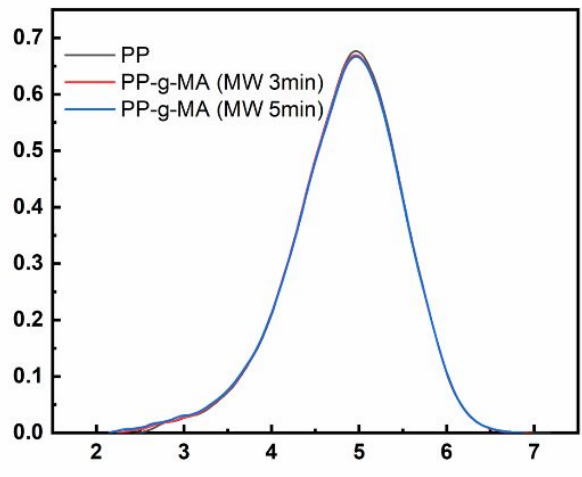

(D)

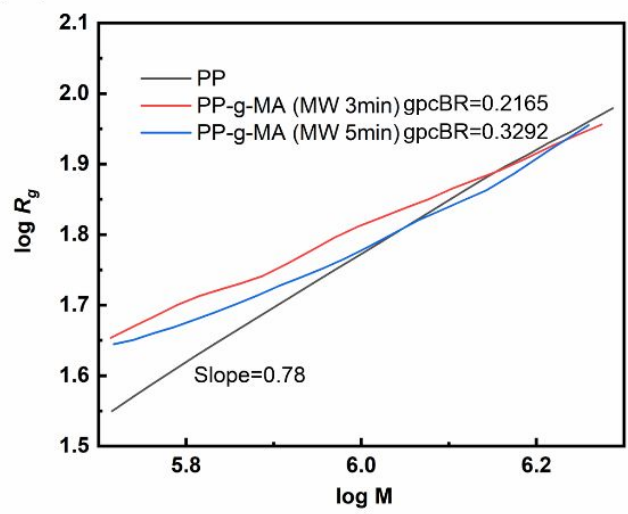

Figure S3. Curves of molecular weight distribution (MWD) and square radius of gyration $\left(R_{g}\right)$ of samples determined by SEC. (A) MWD measured by IR detector, (B) MWD measured by viscometry detector, (C) MWD measured by light scattering detector, (D) The relationship between $\log R_{g}$ and $\log \mathrm{M}$

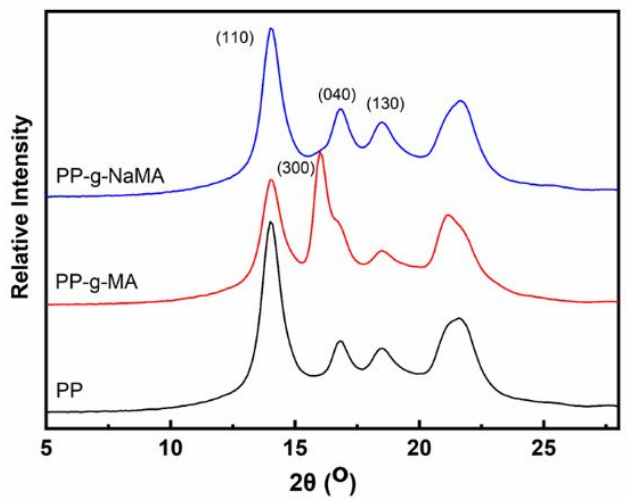

Figure S4. WAXD profiles of PP, PP-g-MA and PP-g-NaMA 
(A)

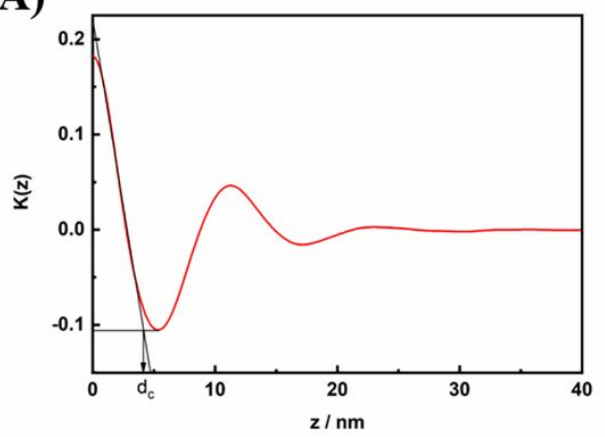

(B)

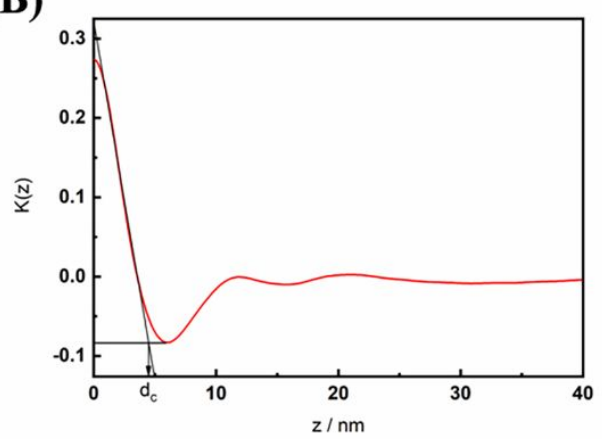

(C)

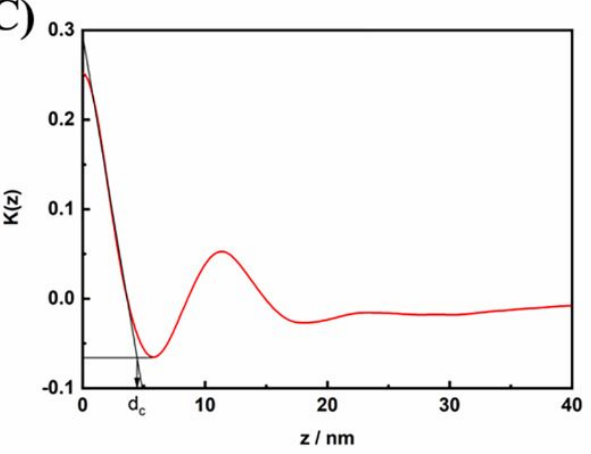

$\left(\mathbf{A}^{\prime}\right)$

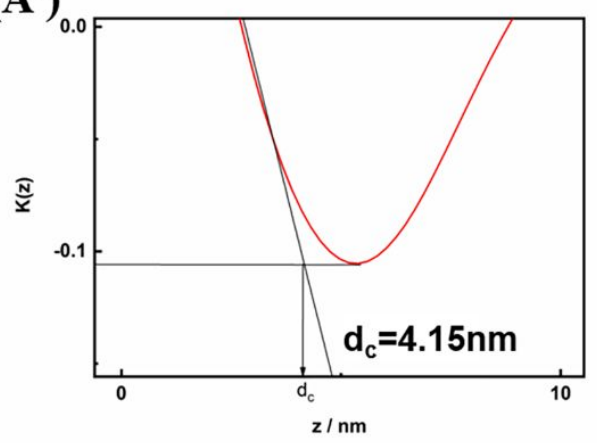

(B')

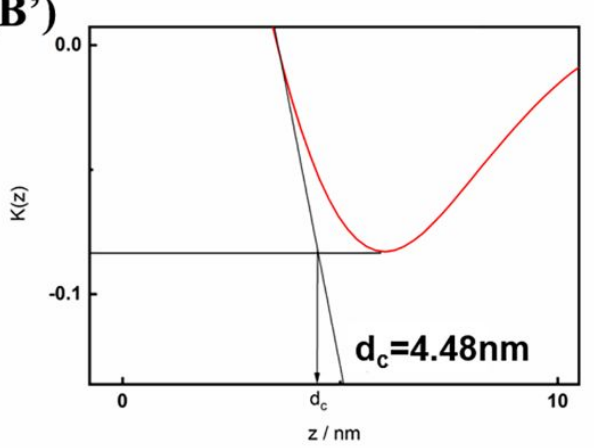

$\left(C^{\prime}\right)$

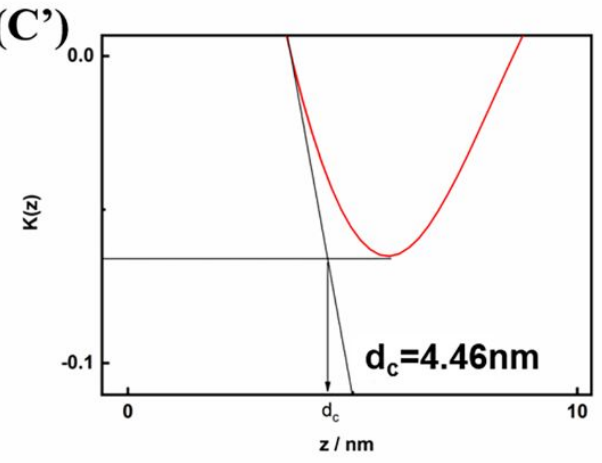

Figure S5. Sketch and enlarged images of one-dimensional correlation function $\mathrm{K}(\mathrm{z})$ for PP (A, A'), PP-g-NaMA (B, B') and PP-g-NaMA (+0.1 MPa) (C, C'). $d_{c}$ is PP lamella thickness. 

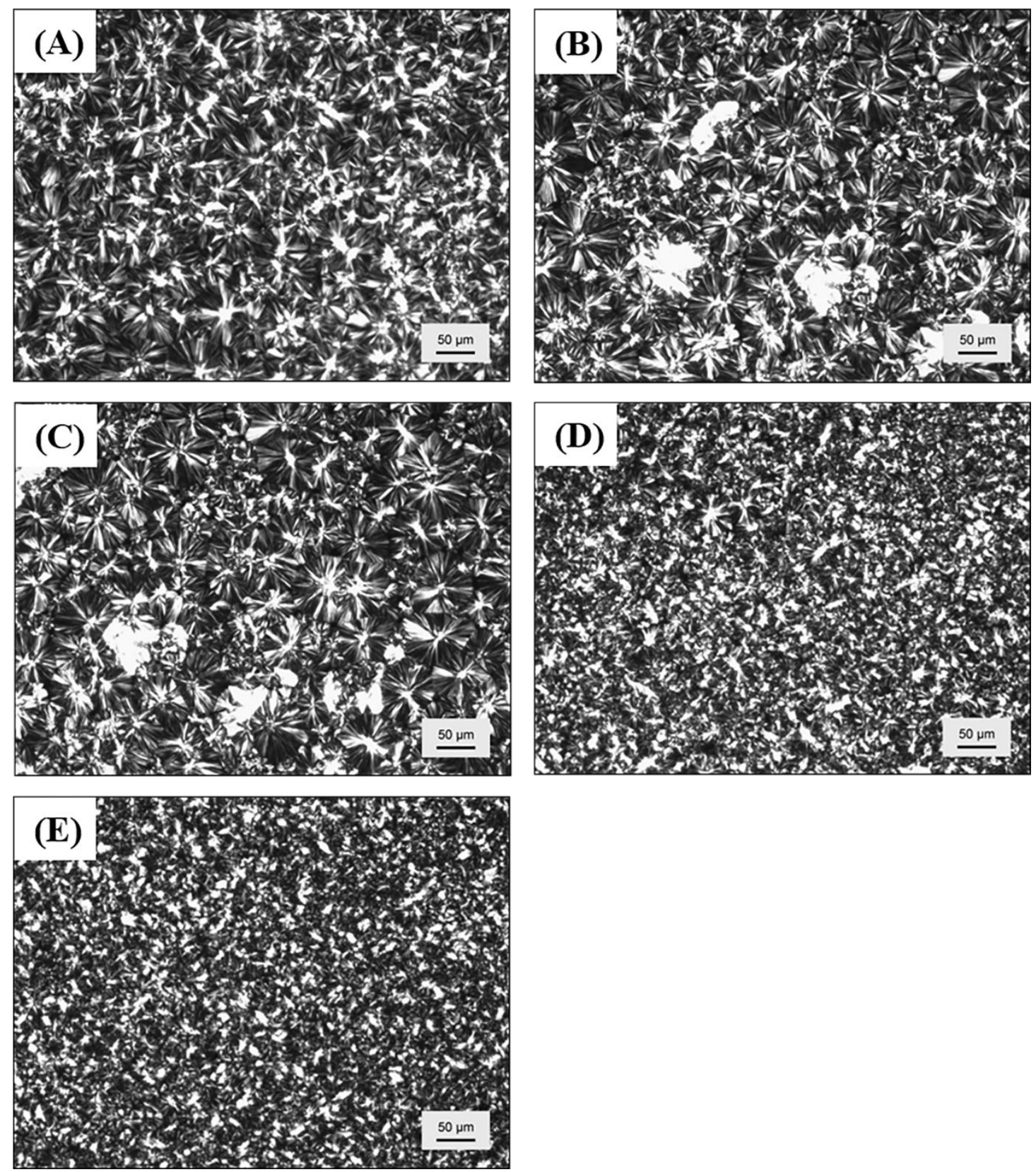

Figure S6. Polarized light microphotographs of (A)PP, (B) PP-g-MA, (C) PP-g-MA (+0.1MPa), (D) PP-g-NaMA, (E) PP-g-NaMA (+0.1MPa). 
(A)

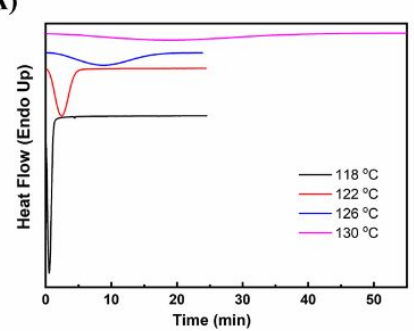

(D)

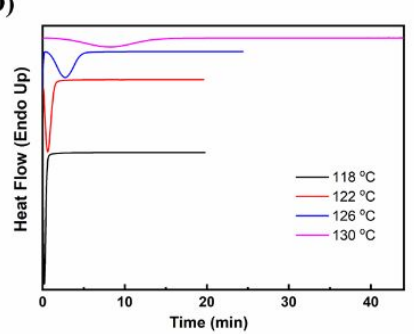

(G)

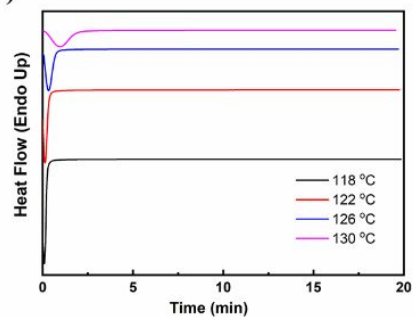

(B)

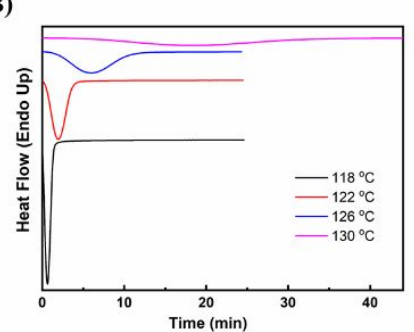

(E)

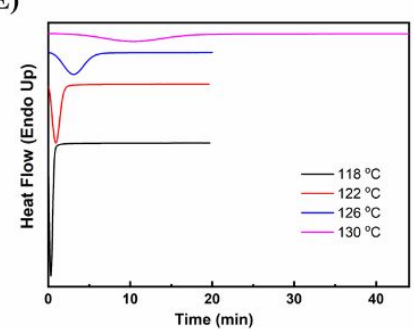

(C)

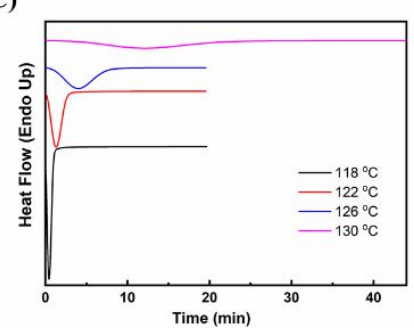

(F)

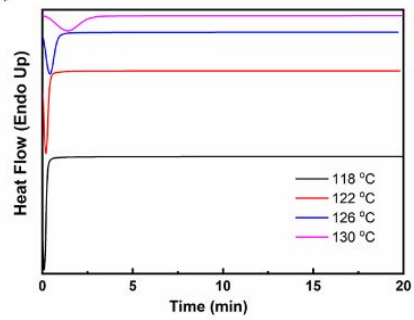

Figure S7. Isothermal crystallization curve of (A)PP, (B) PP-g-NaMA, (C) PP-gNaMA (+0.1MPa), (D) PP-g-NaMA-rGO, ( E ) PP-g-NaMA-rGO (+0.1MPa) , (F)PP $+0.1 \%$ NA11, (G)PP $+0.3 \%$ NA11 at $118{ }^{\circ} \mathrm{C}, 122{ }^{\circ} \mathrm{C}, 126{ }^{\circ} \mathrm{C}$ and $130{ }^{\circ} \mathrm{C}$. 

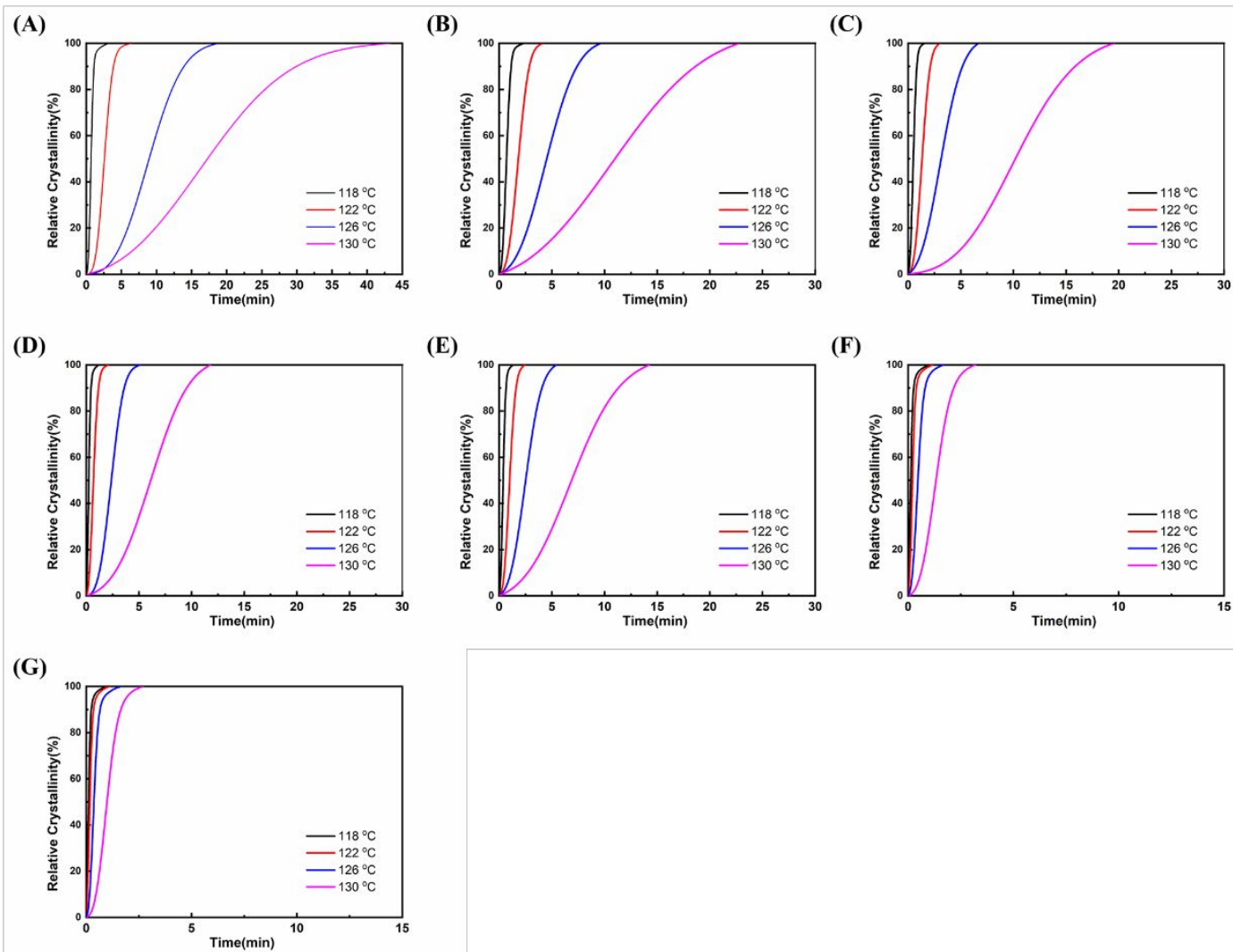

Figure S8. Curve of relative crystallinity Vs time of isothermal crystallization at different temperatures for (A)PP, (B) PP-g-NaMA, (C) PP-g-NaMA (+0.1MPa), (D) PP-g-NaMA-rGO, ( E ) PP-g-NaMA-rGO (+0.1MPa) , (F)PP+0.1\% NA11 and (G)PP+0.3\% NA11. 
(A)

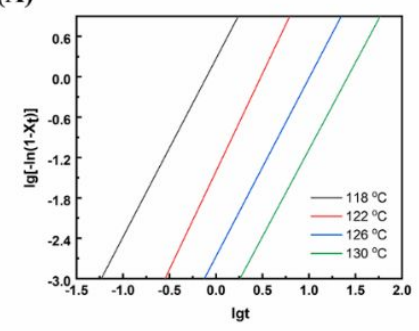

(D)

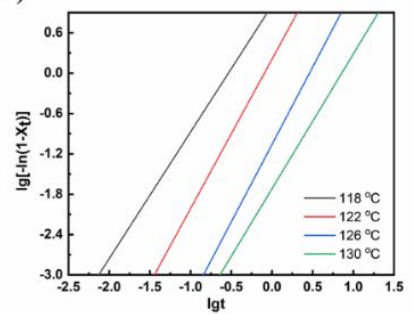

(G)

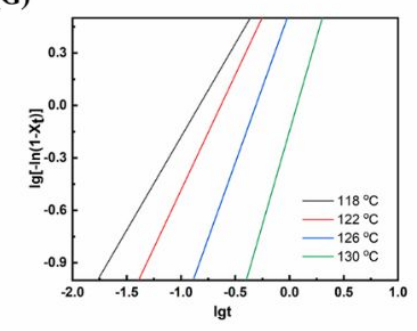

(B)

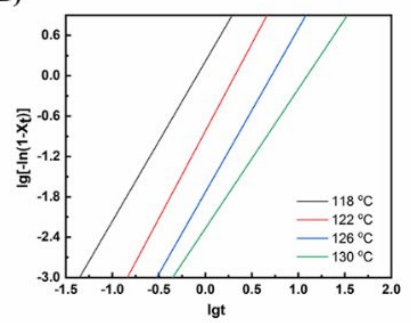

(E)

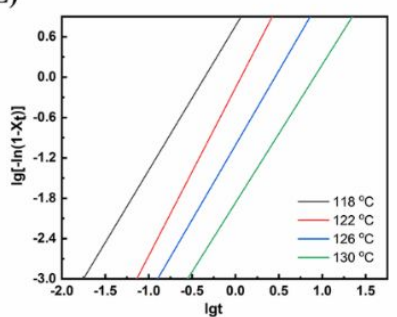

(C)

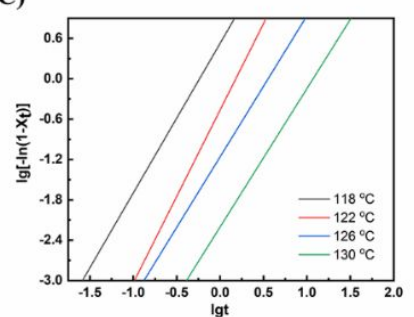

(F)

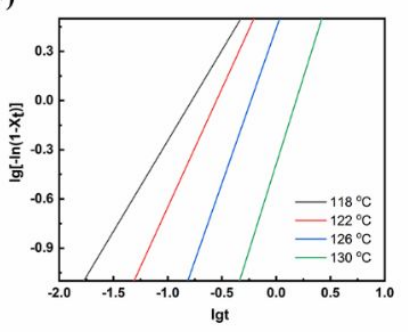

Figure S9. Plots of isothermal crystallization at $118,122,126$ and $130^{\circ} \mathrm{C}$ of

(A)PP, (B) PP-g-NaMA, (C) PP-g-NaMA (+0.1MPa), (D)PP-g-NaMA-rGO,

E) PP-g-NaMA-rGO (+0.1 MPa), (F)PP+0.1\% NA11, (G)PP+0.3\% NA11.

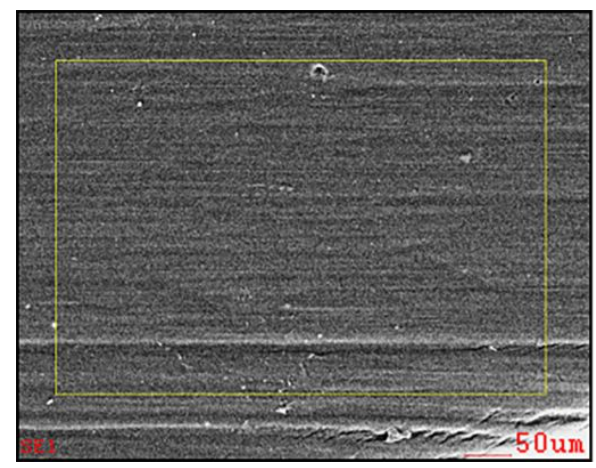

\begin{tabular}{|c|c|c|}
\hline Element & $\mathbf{W t} \%$ & $\mathbf{A t} \%$ \\
\hline $\mathbf{C K}$ & 97.41 & 98.09 \\
\hline $\mathbf{O K}$ & 02.44 & 01.84 \\
\hline SiK & 00.16 & 00.07 \\
\hline Matrix & Correction & ZAF \\
\hline
\end{tabular}

Figure S10. EDS data of PP-g-vinyl trimethoxysilane prepared by NaCl-assisted microwave heating, microwave heating time is $7 \mathrm{~min}$. 
Table S1 Isothermal crystallization kinetic data of PP, PP-g-NaMA and NA11 modified PP samples

\begin{tabular}{|c|c|c|c|c|}
\hline Sample & $T_{c}\left({ }^{\circ} \mathrm{C}\right)$ & $\mathrm{n}$ & $\mathrm{K}\left(\min ^{-n}\right)$ & $t_{1 / 2}(\min )$ \\
\hline \multirow[t]{4}{*}{ PP } & 118 & 2.64 & 1.86 & 0.69 \\
\hline & 122 & 2.71 & 0.04 & 2.69 \\
\hline & 126 & 2.65 & 0.00 & 8.86 \\
\hline & 130 & 2.59 & 0.00 & 22.67 \\
\hline \multirow[t]{4}{*}{ PP-g-NaMA } & 118 & 2.37 & 1.62 & 0.70 \\
\hline & 122 & 2.59 & 0.15 & 1.80 \\
\hline & 126 & 2.43 & 0.02 & 4.43 \\
\hline & 130 & 2.07 & 0.01 & 10.46 \\
\hline PP-g-NaMA & 118 & 2.22 & 3.39 & 0.49 \\
\hline \multirow[t]{3}{*}{$(+0.1 \mathrm{MPa})$} & 122 & 2.56 & 0.35 & 1.31 \\
\hline & 126 & 2.09 & 0.07 & 3.01 \\
\hline & 130 & 2.06 & 0.01 & 9.90 \\
\hline \multirow[t]{4}{*}{ PP-g-NaMA-rGO } & 118 & 2.01 & 10.23 & 0.24 \\
\hline & 122 & 2.21 & 1.62 & 0.68 \\
\hline & 126 & 2.30 & 0.09 & 2.46 \\
\hline & 130 & 2.00 & 0.02 & 6.03 \\
\hline PP-g-NaMA-rGO & 118 & 2.14 & 5.75 & 0.37 \\
\hline$(+0.1 \mathrm{MPa})$ & 122 & 2.49 & 0.69 & 1.00 \\
\hline
\end{tabular}




\begin{tabular}{ccccc} 
& 126 & 2.21 & 0.10 & 2.43 \\
& 130 & 2.05 & 0.01 & 6.83 \\
$\mathrm{PP}+0.1 \%$ NA11 & 118 & 1.10 & 7.23 & 0.12 \\
& 122 & 1.45 & 6.27 & 0.22 \\
& 126 & 1.89 & 2.75 & 0.48 \\
$\mathrm{PP}+0.3 \%$ NA11 & 130 & 2.11 & 0.41 & 1.28 \\
& 118 & 1.07 & 7.68 & 0.10 \\
& 122 & 1.32 & 6.82 & 0.18 \\
& 126 & 1.73 & 3.44 & 0.40 \\
& 130 & 2.14 & 0.71 & 0.99 \\
\hline
\end{tabular}

